\title{
Evolution of microRNA expression during human bronchial squamous carcinogenesis
}

\author{
C. Mascaux*,f, J.F. Laes ${ }^{\#, f}$, G. Anthoine*, A. Haller ${ }^{\circ}$, V. Ninane ${ }^{+}$, \\ A. Burny ${ }^{\S}$ and J.P. Sculier*
}

ABSTRACT: MicroRNAs, negative post-transcriptional regulators of gene expression, are involved in cancer.

Their role in early bronchial carcinogenesis was analysed in 60 biopsies obtained by fluorescence bronchoscopy (six per stage: normal tissue of nonsmokers, normal normofluorescent and hypofluorescent bronchial tissue of smokers, hyperplasia, metaplasia, mild, moderate and severe dysplasia, in situ carcinoma and invasive squamous cell carcinoma (SQCC)).

In total, 69 microRNAs were found to be differentially expressed in the course of bronchial carcinogenesis. Among them, some microRNAs showed a linear evolution of their expression level, such as miR-32 and miR-34c, whose expression progressively decreased from normal bronchial tissues of nonsmokers to SQCC. Others behaved differently at successive stages, such as miR-142-3p or miR-9, or are only altered from a specific stage, such as miR-199a or miR-139. MicroRNAs globally followed a two-step evolution, first decreasing (a reverse of their increase during embryogenesis) during the earliest morphological modifications of bronchial epithelium, and thereafter increasing at later stages of lung carcinogenesis. Moreover, microRNA expression was very efficient for the prediction of the histological classification between low- and high-grade lesions and between in situ and invasive carcinoma.

The present data show, for the first time, that microRNAs are involved in bronchial carcinogenesis from the very early steps of this process and, thus, could provide tools for early detection of lung cancer.

KEYWORDS: Carcinogenesis, lung, lung cancer, microRNAs, premalignant

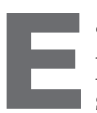
ach year, lung cancer kills 1,300,000 persons worldwide and its incidence is steadily increasing. Improving its cure rate is thus a major public health objective. The bad prognosis of this cancer is mainly explained by the fact that the diagnosis is generally made only at advanced stages. Therefore, identification of early biomarkers, especially if they are detectable by noninvasive methods (serum, sputum, etc.) in highrisk patients (mainly smokers), is mandatory. Lung squamous cell carcinoma (SQCC) is the end-point of a whole range of morphological abnormalities [1] that are diffusely displayed in the bronchial airways of smokers (concept of field cancerisation) and that could be used to identify markers of the ongoing cancer process.

Discovery of microRNAs (miRNAs), negative post-transcriptional regulators of gene expression, was a landmark in molecular biology. Recent studies demonstrated that mutations in
miRNAs or their aberrant expression are associated with diverse human diseases, including cancers. Indeed, miRNAs might act as oncogenes or tumour suppressor genes. Many miRNAs are located on fragile sites and genomic regions involved in cancer [2]. They play important roles in cell differentiation, cell growth and cell death [3]. They have been found to be involved in known oncogenic pathways like those of p53 [4], $\mathrm{Bcl} 2$ [5] or K-Ras [6]. Finally, miRNAs seem to be very significant prognostic factors in patients with different tumours [7-10] and could be useful for treatment [11].

Interestingly, miRNAs maintain, even in cancer, the specificity of their developmental lineage [7]. Expression of miRNAs is tissue and cell-type regulated [12]. Moreover, it has also been shown that, even if different tumour types display highly different patterns of miRNA expression, tumours with a common embryonic precursor
AFFILIATIONS

*Dept of Intensive Care and Thoracic Oncology, and

-Dept of Pathology, Institut Jules Bordet, Centre des Tumeurs de I'Université Libre de Bruxelles, ${ }^{+}$Dept of Pneumology CHU Saint-Pierre, Brussels, \#Microarrays Unit, DNAVision, Gosselies, and

${ }^{\S}$ Laboratory of Molecular and Cellular Biology, Faculté Universitaire des Sciences Agronomiques de Gembloux, Gembloux, Belgium. ${ }^{f}$ Both authors contributed equally to this article.

\section{CORRESPONDENCE}

C. Mascaux

Institut Jules Bordet

Rue Héger-Bordet 1

B-1000 Brussels

Belgium

Fax: 3225343756

E-mail: celine.mascaux@bordet.be

Received:

June 042008

Accepted after revision:

October 012008

SUPPORT STATEMENT

C. Mascaux is a Research Fellow of the FNRS (National Fund of Scientific Research, Brussels, Belgium). This study was supported by grants from the FRSM (Fund of Medical Scientific Research), from the Télévie-FNRS and from "Les Amis de l'Institut Jules Bordet" (all Brussels).

STATEMENT OF INTEREST None declared. 
still express common miRNA patterns $[7,13]$. Therefore, their pattern of expression very efficiently classifies different tumour types in a more robust way than gene expression microarrays [7].

The current authors studied the evolution of miRNA levels of expression in biopsies at the successive stages of human bronchial squamous carcinogenesis in order to determine their potential role in this pathological process and to identify potential tools for early detection of lung cancer.

\section{MATERIAL AND METHODS}

\section{Sample collection and RNA isolation}

All biopsies were obtained between 2003 and 2007 after informed consent from the patients and approval by the local ethical committee (Institut Jules Bordet, Brussels, Belgium). Selected patients had a minimum smoking exposure of 30 pack-yrs and/ or a history of lung or head and neck cancer. Fluorescence bronchoscopy was performed under local anaesthesia and all hypofluorescent areas were biopsied for histopathological diagnosis. Biopsies were classified, using the 1999 histological World Health Organization/International Association for the Study of Lung Cancer classification of pre-invasive and invasive squamous lesions of the bronchus [14], into microscopically normal bronchial epithelium, hyperplasia, metaplasia, mild, moderate and severe dysplasia, carcinoma in situ (CIS) and invasive SQCC. Normal bronchial epithelium was biopsied both in normo- and hypofluorescent areas in smokers and in six nonsmokers with healthy lungs. A total of 60 biopsies were included, with six samples of each category: normal bronchial epithelium of nonsmokers, normal normofluorescent bronchial epithelial tissue of smokers, histologically normal but hypofluorescent bronchial epithelium of smokers, hyperplasia, metaplasia, mild, moderate and severe dysplasia, CIS and SQCC. In each case, two biopsies were taken with a clean forceps in the same area. One biopsy was used for histopathology and the second biopsy was immediately dropped into Tripure Isolation Reagent (Roche Diagnostics, Indianapolis, IN, USA), lysed by ultraturaxing and frozen in liquid nitrogen. All samples were kept at $-80^{\circ} \mathrm{C}$. RNA extraction was performed according to a classical phenol/ chloroform protocol as described by the Tripure provider. As carrier, $20 \mu \mathrm{g}$ of glycogen (Roche Diagnostics) was added, and the separation between the organic and the aqueous phase was performed on Phase Lock Gel (Eppendorf, Hamburg, Germany) to optimise the recovery of nucleic acids. Isolated RNAs were assessed for quantity and purity on the NanoDrop ND-1000 spectrophotometer (NanoDrop Technologies, Rockland, DE, USA) and for quality on the Agilent 2100 bioanalyser with RNA 6000 NanoAssay (Agilent Technologies, Palo Alto, CA, USA). RNAs were stored at $-80^{\circ} \mathrm{C}$.

\section{MiRNA expression analyses}

The miRNAs were retro-transcribed and amplified (by PCR) using the multiplex RT TaqMan MicroRNA Low Density Array (LDA) Assays (Applied Biosystems, Foster City, CA, USA). For each sample, $160 \mathrm{ng}$ of starting total RNA (20 $\mathrm{ng}$ for each of the eight RT-PCRs) was used. The global miRNAs profiling, for 365 human miRNAs, was then performed using the TaqMan LDA Human microRNA Panel v1.0 (Applied Biosystems). All the quality control tests were validated: blanks and reproducibility (standard deviation of cycle threshold $(\mathrm{CT})<1$ ) of the two small nucleolar house-keeping RNAs, RNU48 (SNORD48) and RNU44 (SNORD44). The amount of RNA from each sample was calibrated to the more stable (between the different arrays) small nucleolar house-keeping RNA, RNU48. This value gave a delta CT $(\Delta \mathrm{CT})$ value for each miRNA (miRNA CT value minus RNU48 CT value). The average $\Delta \mathrm{CT}$ s were calculated for each group of six samples and the delta $\Delta \mathrm{CTs}(\Delta \Delta \mathrm{CTs})$, corresponding to the differences between two groups, were obtained by subtracting the average $\Delta \mathrm{CT}$ of the second group from those of the first one. Fold differences were calculated as $2^{(-\Delta \Delta C T)}$, as a decrease in $1 \mathrm{CT}$ value was equivalent to a two-fold increase in the starting amount of cDNA. For the down-regulated miRNAs, it was calculated by $1 / 2^{(-\Delta \Delta C T)}$.

\section{Statistical assessment}

First, unsupervised average linkage hierarchical clustering including all samples was performed to exclude any experimental bias. Secondly, unpaired t-tests were used to assess the statistical significance of the difference in miRNA expression between two groups. In addition, a correction for multiple testing (CMT) was performed according to Bonferroni's family-wise error rate. For both tests, the threshold for statistical significance was $p<0.05$. Finally, in order to assess the discriminating potential of miRNA expression profiles, clustering using centred correlation and average linkage was performed.

\section{RESULTS}

\section{Differentially expressed miRNAs during squamous cell carcinogenesis}

Comparisons of miRNAs between successive stages did not show any statistically significant differences, probably due to the small number of patients included at each stage. Therefore, the nine histological stages were combined in larger groups according to a molecular classification previously performed on the same biopsies. These groups are described in table 1.

First, miRNA expression profiles were compared between normal bronchial epithelium of nonsmokers (group 0) and other groups of biopsies at different stages of squamous carcinogenesis in smokers (groups 1-3). MiRNA expression profiles between successive groups and subgroups in smokers (groups 1, 2, 3, 3A and 3B; table 1) were then successively compared, and comparisons were also made between CIS and SQCC. Eight comparisons were thus successively performed between groups 0 versus 1, 2 or $3 ; 1$ versus $2 ; 2$ versus 3 or $3 \mathrm{~A} ; 3 \mathrm{~A}$ versus $3 \mathrm{~B}$ and CIS versus $3 \mathrm{~B}$. In total, $69 \mathrm{miRNAs}$ were found to be significantly differentially expressed in at least one of the eight comparisons. The names of these 69 miRNAs and the stage(s) at which their expression is significantly modified are described in table 2. In the eight successive comparisons of miRNA expression profiles, six, 19, 19, 16, 30, 15, 20 and 10 miRNAs were found to be significantly differentially expressed. In particular, expression of miR-34c, miR-15a and miR-32 was progressively reduced from group 0 to groups 1,2 and 3. Additionally, 16 of the 69 miRNAs remained significantly differentially expressed in one or more comparison(s) after CMT: zero, three, six, four, five, four, one and two miRNAs at each of the eight successive comparisons.

\section{Two-step evolution of miRNA expression}

As illustrated in table 3, two successive waves were globally observed in miRNA expression profiles during lung squamous 


\begin{tabular}{|c|c|c|c|c|c|c|c|c|c|c|}
\hline Stage & 0 & 1 & 2 & 3 & 4 & 5 & 6 & 7 & 8 & 9 \\
\hline Group & 0 & 1 & 1 & 1 & 2 & 2 & 2 & $3 A^{\#}$ & $3 A^{\#}$ & $3 B^{\#}$ \\
\hline
\end{tabular}

carcinogenesis: first, a downregulation and, secondly, an upregulation of the majority of miRNAs. In the first four comparisons of miRNA profiles, between groups 0 and 1,0 and 2, 0 and 3 , and 1 and 2, the majority of miRNAs were downregulated: $83,84,74$ and $87.5 \%$, respectively. In the later four comparisons, the proportion of downregulated miRNAs decreased and that of upregulated miRNAs increased. This increase in the proportion of upregulated miRNAs began in the comparison between groups 2 and 3 , and became $\geqslant 50 \%$ in the comparisons between groups 2 and $3 \mathrm{~A}, 3 \mathrm{~A}$ and $3 \mathrm{~B}$, and CIS and $3 \mathrm{~B}$.

While some miRNAs were modified at one specific step of lung squamous carcinogenesis only (e.g. miR-199a or miR139), others evolved across successive steps. Among them, some miRNAs showed a two-wave evolution, all being firstly downregulated and then upregulated (miR-142-3p, miR-1425p, miR-9, miR-10b and miR-214).

\section{Sample group classification by miRNA profiles}

Using the miRNAs that were differentially expressed between groups 0,1 and 2, it was not possible to segregate samples $a$ posteriori according their respective groups. However, the miRNAs differentially expressed between group 2 and 3 (fig. 1), as well as between groups 2 and 3A (fig. 2), and CIS and invasive carcinomas (group 3B; fig. 3), were very efficient in enabling the separation and classification of individual samples of these groups. The first and second clustering experiments, based on the differentially expressed miRNAs between groups 2 and 3 or $3 \mathrm{~A}$, show that these miRNAs segregate samples of groups 2 and 3 or $3 \mathrm{~A}$ into two groups and that only three samples of group 3A (two CIS and one SQCC) were misclassified into group 2 (figs 1 and 2). Finally, the list of miRNAs found to be differentially expressed between CIS and invasive carcinoma perfectly discriminates the samples of these groups into two categories according to their histological classification (fig. 3).

\section{DISCUSSION}

The results of the present study showed that several miRNAs were differentially expressed in the course of bronchial carcinogenesis and were very efficient tools to discriminate between the samples, both between low-grade lesions (metaplasia, mild and moderate dysplasia) and high-grade preneoplastic ones (severe dysplasia and CIS), and between in situ and invasive carcinomas. Moreover, two successive steps in miRNA expression evolution were found. Indeed, at the earliest stages of bronchial carcinogenesis, corresponding to the progression from normal epithelium of nonsmokers to phenotypically normal epithelium from smokers, to hyperplasia and until the group of relatively benign morphological abnormal bronchial tissue of smokers (metaplasia and mild and moderate dysplasia), a significant reduction of the expression of the vast majority of miRNAs was observed. However, during the later stages of carcinogenesis (severe dysplasia, CIS and SQCC), even if 74\% of the altered miRNAs also remained downregulated compared with their level of expression in normal bronchial tissue of nonsmokers, the proportion of upregulated miRNAs (43\%) increased compared with their level of expression in the previous group of lesions (metaplasia, mild and moderate dysplasia) and more so $(80 \%)$ from that latter group to the group of severe dysplasia and CIS.

Bronchial carcinogenesis thus appears to be a process with a two-step evolution. This is reflected by the global decrease of miRNA expression observed at the earliest stages of smokeinduced morphological modifications of bronchial epithelium, followed by an increase in upregulated miRNAs at the latter stages of carcinogenesis. It has been postulated that miRNA expression is highly regulated according to the cell's developmental lineage and to its differentiation stage $[3,7,13]$. In agreement with the hypothesis that an increased level of miRNA expression induces cell and tissue differentiation, miRNA downregulation often occurs in cancer where tissue is losing its normal differentiation [7, 15]. Interestingly, the present data illustrate this concept. Globally, in evolution from groups 0 to 2, corresponding to morphological modifications following the loss of the normal differentiation of the normal ciliated and pseudostratified bronchial epithelium that is replaced by a keratinised and metaplastic one, the large majority of miRNAs were downregulated. Among them, the expression of several miRNAs presented an inverse evolution of their level of expression, compared with their evolution during lung embryogenesis. In particular, miR-34c and miR15a, which are upregulated during lung development [16], were progressively downregulated during lung squamous carcinogenesis. MiR-99a, miR-142-3p and miR-142-5p, also upregulated in lung development [16], were first downregulated at the earliest steps of carcinogenesis and secondly upregulated at later ones. MiR-214 and miR-301, downregulated in lung embryogenesis [16], were upregulated at stages of severe dysplasia, CIS and SQCC.

Several miRNAs enlisted in the present study have been previously reported in lung cancer miRNA studies. However, 
TABLE 2 Statistically significantly evolving microRNAs during lung carcinogenesis

\begin{tabular}{|c|c|c|c|c|c|c|c|c|}
\hline \multirow[t]{2}{*}{ MicroRNA name } & \multicolumn{8}{|c|}{ Groups compared } \\
\hline & 0 and 1 & 0 and 2 & 0 and 3 & 1 and 2 & 2 and 3 & 2 and $3 A$ & $3 A$ and $3 B$ & $\mathrm{CIS}$ and $3 \mathrm{~B}$ \\
\hline hsa-miR-7 & & & & & Up 4.11 & Up 3.96 & & \\
\hline hsa-miR-9 & & Down 11.11 & & & Up $17.89^{\#}$ & Up $15.97^{\#}$ & Up 3.05 & \\
\hline hsa-miR-15a & Down 5.00 & Down 6.67 & Down 3.45 & & & & & \\
\hline hsa-miR-17-5p & & & & & & Up 2.15 & & \\
\hline hsa-miR-32 & Down 6.67 & Down 5.88 & Down 4.17 & & & & & \\
\hline hsa-miR-34a & & Down 5.00 & & & & & & \\
\hline hsa-miR-34c & Down 6.67 & Down $14.29^{\#}$ & Down $25.00^{\#}$ & & Down 2.50 & & & \\
\hline hsa-miR-99a & & & & Down 1.96 & Down 1.89 & & & \\
\hline hsa-miR-134 & & & & & Down 2.70 & & & \\
\hline hsa-miR-135a & & Down 4.17 & Down $4.00^{\#}$ & & Down 4.00 & & & \\
\hline hsa-miR-139 & & & & & Down 2.27 & & Down 5.26 & Down $5.88^{\#}$ \\
\hline hsa-miR-142-3p & Down 2.94 & Down 5.56 & & & Up 2.60 & Up 2.50 & Up 2.51 & Up 2.24 \\
\hline hsa-miR-142-5p & Down 2.38 & & & & Up 2.86 & Up 3.36 & & \\
\hline hsa-miR-143 & & & & Down 3.85 & & & & \\
\hline hsa-miR-145 & & & & Down 2.70 & & & & \\
\hline has-miR-183 & & & & & Up 2.66 & & & \\
\hline hsa-miR-190 & & Down 4.76 & & & & & & \\
\hline hsa-miR-193a & & & & Down $8.33^{\#}$ & & & & \\
\hline hsa-miR-193b & & Down 4.17 & & & & & Up 5.66 & \\
\hline hsa-miR-214 & & & & Down $2.00^{\#}$ & & & Up 2.35 & Up 2.67 \\
\hline hsa-miR-216 & & & Down 3.33 & & Down 2.63 & Down 2.44 & & \\
\hline hsa-miR-218 & & Down 5.56 & Down 3.57 & & & & & \\
\hline hsa-miR-224 & & Up 2.58 & Up $1.63^{\#}$ & Up 3.79\# & Up 1.63 & & & \\
\hline hsa-miR-301 & & Down 3.57 & & & Up 2.47 & & & \\
\hline hsa-miR-324-5p & & Down 10.00 & & & & & & \\
\hline hsa-miR-338 & & & & & & & Up 5.07 & \\
\hline hsa-miR-361 & & Down 6.25 & Down 4.76 & & & & & \\
\hline hsa-miR-362 & & & & & Up 2.75 & Up 3.36 & & \\
\hline hsa-miR-374 & & & Down 1.49 & & & & & \\
\hline hsa-miR-375 & & & Down 6.25 & & Down 7.14 ${ }^{\#}$ & & Down 11.11 & \\
\hline hsa-miR-382 & & & & Down 2.44 & & & & \\
\hline hsa-miR-410 & & & & Down 2.63 & & & & \\
\hline hsa-miR-411 & & & & Down 4.76 & & & & \\
\hline hsa-miR-422b & & & Down 4.76 & & Down 4.76 & Down 4.17 & & \\
\hline hsa-miR-432 & & & & & & & Up 3.46 & \\
\hline hsa-miR-449 & & Down 3.33 & Down 5.56 & & Down 5.56 & & & \\
\hline hsa-miR-449b & & & Down 11.11 & & & & & \\
\hline hsa-miR-451 & & & & & & & & Down 8.33 \\
\hline hsa-miR-452 & & & Up $14.81^{\#}$ & & Up $6.47^{\#}$ & Up $6.10^{\#}$ & & \\
\hline hsa-miR-486 & & & & & & & Down 10.00 & \\
\hline
\end{tabular}




\begin{tabular}{|c|c|c|c|c|c|c|c|c|}
\hline hsa-miR-487b & & & & & & & Up 8.19 & Up $19.03^{\#}$ \\
\hline hsa-miR-492 & & & & & Down 2.5 & & & \\
\hline hsa-miR-545 & & & & Down 2.78 & & & & \\
\hline hsa-miR-565 & & & & & Down 1.67 & & & Down 2.08 \\
\hline hsa-miR-572 & & & & & Down 3.45 & & & Down 3.85 \\
\hline hsa-miR-597 & & & & & & & Up 5.43 & \\
\hline hsa-miR-615 & & & & & & & & Up 9.74 \\
\hline hsa-miR-622 & & & & & & & Down 6.25 & \\
\hline hsa-miR-650 & & & & & & Up 4.97 & & \\
\hline hsa-miR-659 & Up 4.83 & & & Down 3.70 & & & Up 6.81 & \\
\hline
\end{tabular}

Data are presented as fold changes. CIS: carcinoma in situ; down: downregulated; up: upregulated. All the microRNAs found differentially expressed in at least one of the eight comparisons are shown (t-test $\mathrm{p}<0.05$ ). ${ }^{*}$ : microRNAs remaining statistically differentially expressed in a specific comparison after correction for multiple testing (CMT) at Bonferroni corrected $p$-value $<0.05$. In the first comparison (group 0 versus group 1), no microRNA expression difference remained statistically significant after CMT, whereas, in the seven other comparisons, the numbers of microRNAs with differential expression remaining statistically significant after CMT were three, six, four, five, four, one and two (from left to right)

none of the five miRNAs recently reported as prognostic factors by $\mathrm{Yu}$ et al. [10] was detected in the current study. Ethnic differences, the study by Yu et al. [10] involving exclusively Chinese patients, perhaps explain these discrepancies. Two miRNAs upregulated in the current study of lung carcinogenesis, miR-224 and miR-214, and one downregulated, miR-32, were reported in a study by YANAIHARA et al. [9], but inversely for miR-224, which was downregulated at invasive stages [9], perhaps in accordance with the two-steps model of bronchial carcinogenesis. MiR-9, downregulated in a series of lung cancers with a higher proportion of adenocarcinomas [9], was also downregulated at early stages in the current study, from normal tissue of nonsmokers to group 2, and thereafter upregulated, from the latter stages to group $3 \mathrm{~A}$ and again to group $3 \mathrm{~B}$. This pattern is another example of the two-step evolution observed for miRNA expression levels during bronchial carcinogenesis.
MiR-203, the most significantly upregulated miRNA in group 2 compared with both normal bronchial tissues of nonsmokers and of smokers, was previously reported to be increased in lung cancer A549 cell lines [17] and in patients [9]. Finally, miR-17-5p, upregulated at the level of group 3A, belongs to the miR-17-92 oncogenenic polycistron [18], which is overexpressed in cancer and, in particular, in lung cancer [19], where it enhances cell proliferation and inhibits cell differentiation.

Some miRNAs identified in the present study have been described as related to known oncogenes or tumour suppressor genes, and their expression level evolved through all successive stages from normal bronchi of nonsmokers to invasive SQCC in smokers. In particular, the expression level of miR-34c progressively decreased from normal epithelium of nonsmokers to invasive bronchial lesions of smokers. This

\section{TABLE 3 Two-step evolution of microRNAs in lung squamous carcinogenesis}

\section{Differentially expressed} microRNAs

\begin{tabular}{lcccccccc}
\hline & $\mathbf{0}$ and $\mathbf{1}$ & $\mathbf{0}$ and $\mathbf{2}$ & $\mathbf{0}$ and $\mathbf{3}$ & $\mathbf{1}$ and $\mathbf{2}$ & $\mathbf{2}$ and $\mathbf{3}$ & $\mathbf{2}$ and $\mathbf{3 A}$ & $\mathbf{3 A}$ and $\mathbf{3 B}$ & $\mathbf{C I S}$ and $\mathbf{3 B}$ \\
Total number & 6 & 19 & 19 & 16 & 30 & 15 & 20 & 10 \\
Downregulated & $5(83)$ & $16(84)$ & $14(74)$ & $14(87.5)$ & $17(57)$ & $3(20)$ & $8(40)$ & $5(50)$ \\
Upregulated & $1(17)$ & $3(16)$ & $5(26)$ & $4(12.5)$ & $13(43)$ & $12(80)$ & $12(60)$ & $5(50)$ \\
\hline
\end{tabular}

Data are presented as $\mathrm{n}$ or $\mathrm{n}(\%)$. CIS: carcinoma in situ. Two successive waves were observed in microRNA expression profiles during lung squamous carcinogenesis: first, a downregulation and, secondly, an upregulation of microRNAs. In the first four comparisons of microRNA profiles, between groups 0 and 1,0 and 2,0 and 3 , and 1 and 2, the large majority of microRNAs were downregulated. In the later four comparisons, the proportion of downregulated microRNAs decreased and that of upregulated microRNAs increased, with $\geqslant 50 \%$ being upregulated for comparisons between groups 2 and $3 \mathrm{~A}, 3 \mathrm{~A}$ and $3 \mathrm{~B}$, and $\mathrm{CIS}$ and $3 \mathrm{~B}$. 


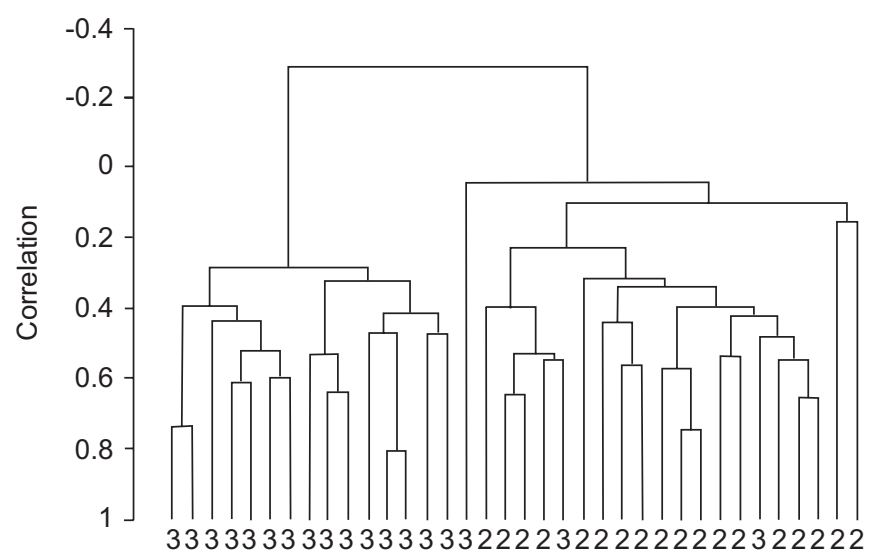

FIGURE 1. Classification of samples from groups 2 and 3, based on microRNAs differentially expressed between these groups. Dendrogram for clustering experiments, using centred correlation and average linkage. The dendrogram, based on the differentially expressed microRNAs between groups 2 (metaplasia, mild and moderate dysplasia) and 3 (severe dysplasia, carcinoma in situ (CIS) and invasive carcinoma), segregated samples of groups 2 and 3 into two groups. Only three samples of group 3 (two severe dysplasias and one CIS) were misclassified into group 2.

miRNA was recently identified as a transcriptional target for the tumour suppressor p53 [4]. The progressive downregulation of miR-34c therefore reflects the progressive loss of the protective activity of p53 and is in accordance with previous studies reporting p53 alterations starting in histologically normal tissue and hyperplasia of smokers [20]. MiR-15a was also progressively downregulated. First described in chronic lymphocytic leukaemia [21], miR-15a has tumour suppressor properties through the inhibition of the anti-apoptotic $\mathrm{Bcl} 2$ protein, which is involved in lung carcinogenesis [5].

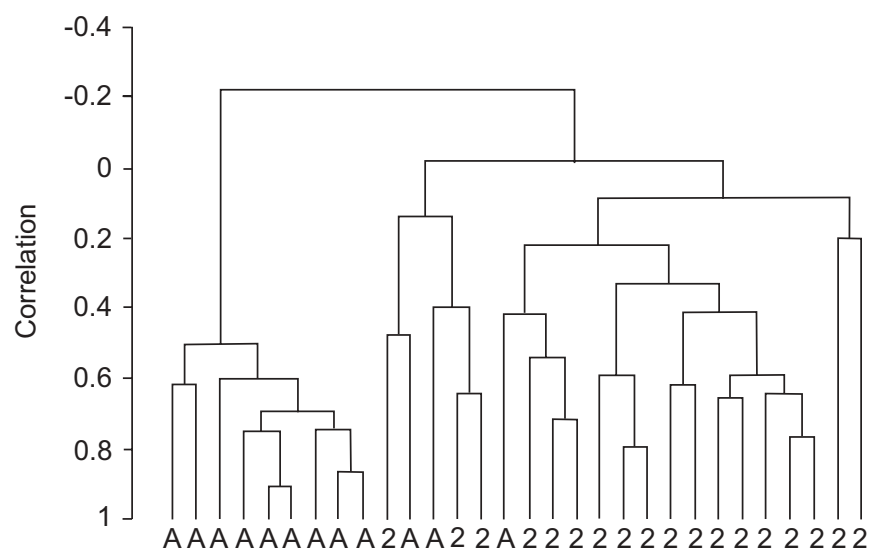

FIGURE 2. Classification of samples from groups 2 and $3 A(A)$, based on microRNAs differentially expressed between these groups. Dendrogram for clustering experiments, using centred correlation and average linkage. The dendrogram, based on the differentially expressed microRNAs between groups 2 (metaplasia, mild and moderate dysplasia) and 3A (severe dysplasia and carcinoma in situ (CIS)), segregated samples of groups 2 and $3 \mathrm{~A}$ into two groups. Only three samples of group $3 \mathrm{~A}$ (two severe dysplasias and one CIS) were misclassified into group 2.

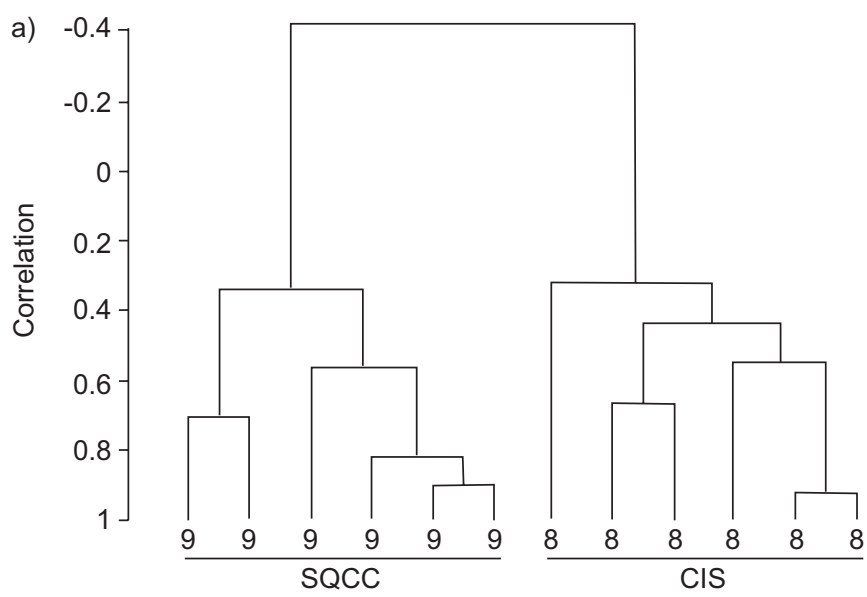

b)

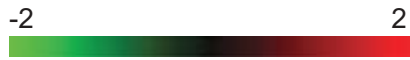

Log intensities

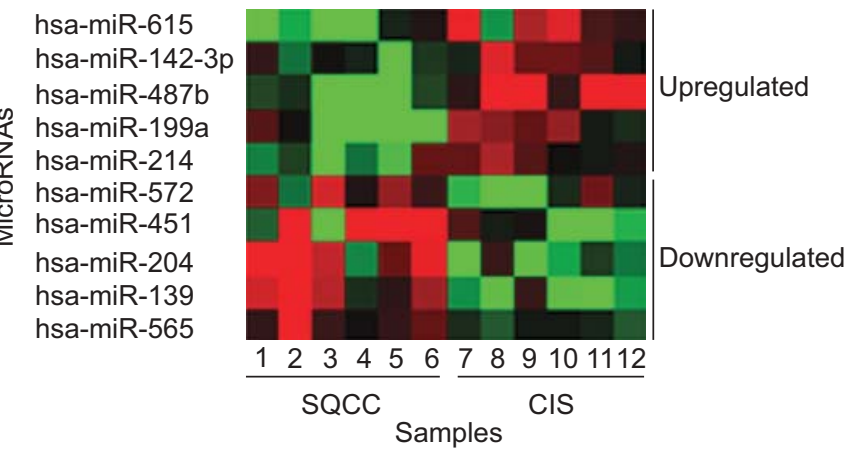

FIGURE 3. Classification of samples between carcinoma in situ (CIS; stage 8) and invasive squamous cell carcinoma (SQCC; stage 9), based on microRNAs differentially expressed between these groups. a) Dendrogram for clustering experiments, using centred correlation and average linkage. MicroRNAs differentially expressed between CIS and SQCC segregated the samples of these stages into two groups according to their histological classification: the six samples of stage 8 and of stage 9 were well separated into two different branches. b) The log intensities are directly proportional to the cycle threshold (CT), and the green (smaller CT) and the red (higher CT) represent up- and downregulated microRNAs, respectively. The first five microRNAs were upregulated in SQCC (the first six samples) compared with CIS (the last six samples). The last five microRNAs were downregulated from CIS to SQCC.

Other miRNAs enlisted in the current study have not yet been reported to be related to lung cancer but have been described in other cancers: miR-196a in pancreatic carcinogenesis [22]; miR-487b in Burkitt lymphoma [23]; miR-204 as an inhibitor of apoptosis and reported in acute lymphocytic leukaemias [24]; miR-106b in hepatocellular carcinomas [25]; and miR-199a in ovarian cancer [26] and in acute myeloid leukaemia, where it is a signature for a bad prognosis [27]. Conversely, several miRNAs identified in the present study, such as miR-452, miR196b, miR-375, miR-193a and miR-139, have not been reported to be associated with any type of cancer until now, and their function needs to be determined.

Finally, the present study of the evolution of miRNA expression in bronchial pre-invasive lesions aimed to identify early biomarkers, especially ones detectable by noninvasive methods 
(serum, sputum, etc.) in high-risk patients, for early detection of lung cancer. To the current authors' knowledge, no tests or biomarkers are routinely available to solve this problem. The only attempt to identify a molecular pattern for pre-neoplastic bronchial lesions using a high-throughput technique was performed by RAHMAN et al. [28], who used a proteomic mass spectrometry approach called MALDI MS (matrix-assisted laser desorption ionisation mass spectrometry). Invasive lung cancer was correctly classified but they could not validate their results for pre-neoplastic lesions. Potential explanations given by the authors were the lack of true normal epithelium for reference, heterogeneity of the sources of analysed tissues, technical problems related to the MALDI MS method and a small number of available pre-neoplastic lesions. Most of these problems were avoided or solved in the present study. It was not possible to assess the prognostic value of miRNA expression in the biopsies because lesions were resected and cured by endoscopic procedure when necessary. However, previously published data indicate that severe dysplasia and CIS are at higher risk of pejorative evolution than lower-grade lesions (32$87 \%$ versus 2-9\%, respectively) [29-31]. Analysis of the current data revealed miRNA signatures differentiating high-grade lesions (severe dysplasia and higher grades) from lower-grade ones. If confirmed by internal and external validity studies and after assessment by more adequate techniques for screening, such as sputum examination, the current observations could have potential important practical implications for the early detection of bronchial lesions requiring treatment. This miRNA signature thus provides an original and promising tool for new lung cancer diagnosis research.

In conclusion, the present study is the first to assess microRNA expression during the successive steps of bronchial carcinogenesis on human biopsies. Most microRNA expression levels evolved in two steps during bronchial carcinogenesis. Initially decreasing during the earliest morphological modifications and the aberrant differentiation of normal bronchial epithelium into a keratinised metaplastic one, they were thereafter found to increase when malignant transformation was engaged at the stage of severe dysplasia. Several microRNAs involved in human lung development showed an inverse evolution of their level of expression during bronchial carcinogenesis, such as $\mathrm{miR}-34 \mathrm{c}$, a transcriptional target of p53, which is upregulated in lung formation, but was inversely and progressively downregulated during the whole process of bronchial carcinogenesis. However, the functional roles of the majority of upregulated or downregulated microRNAs found in the current study remain unknown. These questions require further experiments. Finally, the microRNA patterns of expression were able, remarkably, to segregate high-grade pre-neoplastic lesions from the lower-grade ones, and also carcinoma in situ from invasive carcinoma. The microRNAs differentially expressed between these stages are therefore potential tools for early detection of lung cancer, at preinvasive stages, before the spreading of micrometastases.

\section{ACKNOWLEDGEMENTS}

The authors would like to thank P. Pierard and J. Faber (Institut Jules Bordet and CHU Saint-Pierre, Brussels, Belgium), M. Bruyneel, S. Bensliman and O. Bauwens (all CHU Saint-Pierre), who performed fluorescence bronchoscopy; and C. Decoster
(Institut Jules Bordet and CHU Saint-Pierre), J-F. Godart (no affiliation), B. Martin and Y. Cleuter (both Institut Jules Bordet) for their help in this project. They also thank all patients who participated in this study.

\section{REFERENCES}

1 Auerbach O, Hammond EC, Garfinkel L. Changes in bronchial epithelium in relation to cigarette smoking, 1955-1960 vs 1970-1977. N Eng J Med 1979; 300: 381-385.

2 Calin GA, Sevignani C, Dumitru CD, et al. Human microRNA genes are frequently located at fragile sites and genomic regions involved in cancers. Proc Natl Acad Sci USA 2004; 101: 2999-3004.

3 Hwang HW, Mendell JT. MicroRNAs in cell proliferation, cell death, and tumorigenesis. Br J Cancer 2006; 94: 776-780.

4 Corney DC, Flesken-Nikitin A, Godwin AK, Wang W, Nikitin AY. MicroRNA-34b and microRNA-34c are targets of p53 and cooperate in control of cell proliferation and adhesion-independent growth. Cancer Res 2007; 67: 8433-8438.

5 Cimmino A, Calin GA, Fabbri M, et al. MiR-15 and miR-16 induce apoptosis by targeting BCL2. Proc Natl Acad Sci USA 2005; 102: 13944-13949.

6 Johnson SM, Grosshans H, Shingara J, et al. RAS is regulated by the let-7 microRNA family. Cell 2005; 120: 635-647.

$7 \mathrm{Lu} \mathrm{J}$, Getz G, Miska EA, et al. MicroRNA expression profiles classify human cancers. Nature 2005; 435: 834-838.

8 Volinia S, Calin GA, Liu CG, et al. A microRNA expression signature of human solid tumors defines cancer gene targets. Proc Natl Acad Sci USA 2006; 103: 2257-2261.

9 Yanaihara N, Caplen N, Bowman E, et al. Unique microRNA molecular profiles in lung cancer diagnosis and prognosis. Cancer Cell 2006; 9: 189-198.

$10 \mathrm{Yu}$ SL, Chen HY, Chang GC, et al. MicroRNA signature predicts survival and relapse in lung cancer. Cancer Cell 2008; 13: 48-57.

11 Czech MP. MicroRNAs as therapeutic targets. N Engl J Med 2006; 354: 1194-1195.

12 Wienholds E, Kloosterman WP, Miska E, et al. MicroRNA expression in zebrafish embryonic development. Science 2005; 309: 310-311.

13 Meltzer PS. Cancer genomics: small RNAs with big impacts. Nature 2005; 435: 745-746.

14 Travis WD, Colby TV, Corrin B, Shimosato Y, Brambilla E. World Health Organization International Histological Classification of Tumors. Histological Typing of Lung and Pleural Tumors. 3rd Edn. Berlin, Springer-Verlag, 1999.

15 Cummins JM, Velculescu VE. Implications of micro-RNA profiling for cancer diagnosis. Oncogene 2006; 25: 6220-6227.

16 Williams AE, Moschos SA, Perry MM, Barnes PJ, Lindsay MA. Maternally imprinted microRNAs are differentially expressed during mouse and human lung development. Dev Dyn 2007; 236: 572-580.

17 Cheng AM, Byrom MW, Shelton J, Ford LP. Antisense inhibition of human miRNAs and indications for an involvement of miRNA in cell growth and apoptosis. Nucleic Acids Res 2005; 33: 1290-1297.

$18 \mathrm{He}$ L, Thomson JM, Hemann MT, et al. A microRNA polycistron as a potential human oncogene. Nature 2005; 435: 828-833. 
19 Hayashita $\mathrm{Y}$, Osada $\mathrm{H}$, Tatematsu $\mathrm{Y}$, et al. A polycistronic microRNA cluster, miR-17-92, is overexpressed in human lung cancers and enhances cell proliferation. Cancer Res 2005; 65: 9628-9632.

20 Martin B, Verdebout JM, Mascaux C, et al. Expression of p53 in preneoplastic and early neoplastic bronchial lesions. Oncol Rep 2002; 9: 223-229.

21 Calin GA, Liu CG, Sevignani C, et al. MicroRNA profiling reveals distinct signatures in $\mathrm{B}$ cell chronic lymphocytic leukemias. Proc Natl Acad Sci USA 2004; 101: 11755-11760.

22 Szafranska AE, Davison TS, John J, et al. MicroRNA expression alterations are linked to tumorigenesis and non-neoplastic processes in pancreatic ductal adenocarcinoma. Oncogene 2007; 26: 4442-4452.

23 Beck-Engeser GB, Lum AM, Huppi K, Caplen NJ, Wang BB, Wabl M. Pvt1-encoded microRNAs in oncogenesis. Retrovirology 2008; 5: 4.

24 Tanzer A, Stadler PF. Molecular evolution of a microRNA cluster. J Mol Biol 2004; 339: 327-335.
25 Yang J, Zhou F, Xu T, et al. Analysis of sequence variations in 59 microRNAs in hepatocellular carcinomas. Mutat Res 2008; 638: 205-209.

26 Iorio MV, Visone R, Di Leva G, et al. MicroRNA signatures in human ovarian cancer. Cancer Res 2007; 67: 8699-8707.

27 Garzon R, Volinia S, Liu CG, et al. MicroRNA signatures associated with cytogenetics and prognosis in acute myeloid leukemia. Blood 2008; 111: 3183-3189.

28 Rahman SM, Shyr Y, Yildiz PB, et al. Proteomic patterns of preinvasive bronchial lesions. Am J Respir Crit Care Med 2005; 172: 1556-1562.

29 Bota S, Auliac JB, Paris C, et al. Follow-up of bronchial precancerous lesions and carcinoma in situ using fluorescence endoscopy. Am J Respir Crit Care Med 2001; 164: 1688-1693.

30 Breuer RH, Pasic A, Smit EF, et al. The natural course of preneoplastic lesions in bronchial epithelium. Clin Cancer Res 2005; 11: 537-543.

31 Venmans BJ, van Boxem TJ, Smit EF, Postmus PE, Sutedja TG. Outcome of bronchial carcinoma in situ. Chest 2000; 117: 1572-1576. 